\title{
Deviations from universality in the fluctuation behavior of a heterogeneous complex system reveal intrinsic properties of components: The case of the international currency market
}

\author{
Abhijit Chakraborty, Soumya Easwaran and Sitabhra Sinha \\ The Institute of Mathematical Sciences, CIT Campus, Taramani, Chennai 600113, India.
}

(Dated: November 8, 2018)

\begin{abstract}
Identifying behavior that is relatively invariant under different conditions is a challenging task in far-from-equilibrium complex systems. As an example of how the existence of a semi-invariant signature can be masked by the heterogeneity in the properties of the components comprising such systems, we consider the exchange rate dynamics in the international currency market. We show that the exponents characterizing the heavy tails of fluctuation distributions for different currencies systematically diverge from a putative universal form associated with the median value $(\simeq 2)$ of the exponents. We relate the degree of deviation of a particular currency from such an "inverse square law" to fundamental macroscopic properties of the corresponding economy, viz., measures of per capita production output and diversity of export products. We also show that in contrast to uncorrelated random walks exhibited by the exchange rate dynamics for currencies belonging to developed economies, those of the less developed economies show characteristics of subdiffusive processes which we relate to the anti-correlated nature of the corresponding fluctuations. Approaches similar to that presented here may help in identifying invariant features obscured by the heterogeneous nature of components in other complex systems.
\end{abstract}

\section{INTRODUCTION}

The discovery that systems at equilibrium exhibit universality near a phase transition has been a path-breaking achievement of statistical physics in the previous century [1]. However, despite considerable effort, fluctuation behavior in biological and socio-economic systems that are far from equilibrium are not yet well understood [2]. Indeed, strong evidence for universality of nonequilibrium transitions is still lacking [3]. The large diversity seen in non-equilibrium critical phenomena poses a major challenge for those trying to uncover general principles underlying the collective dynamics of complex systems occurring in nature and society. Such systems, apart from comprising a large number of interacting components, are often characterized by a large degree of heterogeneity in the properties of individual elements. For example, components of a complex system may exhibit qualitatively distinct dynamics. The local connection density among the elements in different parts may also greatly differ. It is known that such heterogeneity can result in deviation from universal behavior expected near phase transitions [4].

A prototypical example of a complex system with a highly heterogeneous composition is the de-centralized international trade in foreign exchange (FOREX) which constitutes the largest financial market in the world in terms of volume [5]. An advantage of studying its fluctuation behavior over that of other complex systems with many degrees of freedom is the availability of large quantities of high-resolution digital data that are relatively easily accessible for analysis [6]. The different currencies that are traded in the market are each subject to multifarious influences, e.g., related to geographical, economic, political or commercial factors, which can affect them in many different ways. Such a highly heteroge- neous system provides a stark contrast to the relatively simpler systems having homogeneous composition that have typically been investigated by physicists. In particular, we can ask whether the components of a heterogeneous complex system can be expected to show universal features, i.e., phenomena independent of microscopic details, which may potentially be explained using tools of statistical physics. For the specific case of the FOREX market, establishing any robust empirical regularity will be an important contribution towards understanding the underlying self-organizing dynamics in such systems. Note that the domain of microeconomics that is concerned with the dynamics of single markets has seen accumulating evidence suggestive of universality [7]. The most robust of these relate to the nature of the heavytailed distributions of fluctuations in individual stock prices, as well as, equity market indices 8-11], often referred to as the "inverse cubic law" [12, 13]. In contrast, macroeconomic processes have a relative paucity of such "stylized facts". Although the distribution of fluctuations in the exchange rates of currencies has been the subject of several earlier investigations [14, 15], some of which have indeed reported heavy tails for different currencies, there is little agreement concerning the values of the power-law exponents characterizing such tails - not even whether they lie outside the Levy-stable regime 16 19]. This suggests that the nature of the fluctuation distribution for a particular currency could be related to some intrinsic properties of the underlying economy.

In this paper we show that there is indeed a systematic deviation from a putative universal signature - which we refer to as "inverse square law" - for the fluctuation behavior of different currencies depending on two key macroeconomic indicators, viz., the gross domestic product (GDP) per capita related to the economic performance, and the Theil index that measures the diversity of 
exports of the corresponding countries (see data description for details). Thus, several underdeveloped (frontier) economies exhibit currency fluctuations whose distributions appear to be of a Levy-stable nature, while those of most developed economies fall outside this regime. The median value of the exponents quantifying the heavytailed nature of the cumulative fluctuation distributions for all the currencies occur close to 2, i.e., at the boundary of the Levy-stable regime. Our study demonstrates how robust empirical regularities in complex systems can be uncovered when they are masked by the intrinsic heterogeneity among the individual components. We have also characterized the distinct nature of the exchange rate dynamics of different currencies by considering their selfsimilar scaling behavior. Our analysis reveals that while currencies of developed economies follow uncorrelated random walks, those of emerging and frontier economies exhibit sub-diffusive (or mean-reverting) dynamics.

\section{DATA DESCRIPTION}

The data-set we have analyzed comprises the daily exchange rates with respect to the US Dollar (USD) of $N=75$ currencies (see Table \) for the period October 23, 1995 to April 30, 2012, corresponding to $\tau=6035$ days. The rate we use is the midpoint value, i.e., the average of the bid and ask rates for 1 USD against a given currency. The data is obtained from a publicly accessible archive of historical interbank market rates maintained by the Oanda corporation, an online currency conversion site 20] that is used by major corporations, tax authorities and auditing firms worldwide. The interbank (or spot) rate for a currency is the official rate quoted in the media and that apply to large transactions of $10^{6} \mathrm{USD}$ or higher (typically taking place between banks and financial institutions). For each day, the site records an average value that is calculated over all rates collected over a 24 hour period from frequently updated sources in the global foreign exchange market, including online currency trading platforms, leading market data vendors, and contributing financial institutions. We have chosen USD as the base currency for the exchange rate as it is the preferred currency for most international transactions and remains the reserve currency of choice for most economies 21, 22]. We have verified that using other base currencies lead to qualitatively similar fluctuation distributions for exchange rates.

The choice of currencies used in our study is mainly

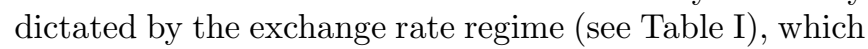
is obtained from the site [20] where we collected the exchange rates data and supplemented by information from the site of another online FOREX services company [23]. In particular, we have not considered currencies whose exchange rate with respect to USD is constant over time. Most of the currencies in our database are floating, either freely under the influence of market forces or managed to an extent with no pre-determined path. Among the re- maining currencies, a few are pegged to USD or some other important currency (such as EUR), but with some variation within a band (which may either be fixed or moving in time). Note that as the EUR was introduced in January 1, 1999, i.e., within the time interval considered by us, we have used the exchange rate for the ECU (European Currency Unit) for the period October 23, 1995 to December 31, 1998.

To ensure that the observed differences in the nature of the fluctuation distributions of currencies is not just a trivial outcome of the different exchange rate regimes, we have performed a two-sample Kolmogorov-Smirnov test 24] with the null hypothesis that the pegged and floating currencies are sampled from the same continuous distribution. A measured $p$-value of 0.39 indicates that the null hypothesis cannot be rejected at $5 \%$ level of significance. We also carried out a Wilcoxon rank sum test 25] with the null hypothesis that both pegged and floating currencies are sampled from continuous distributions with equal medians. We obtained a $p$-value of 0.27 , again indicating that there is not enough evidence to reject the null hypothesis at $5 \%$ level of significance. We thus conclude that the distinct behavior of the currencies in terms of the distribution of their exchange rate returns cannot be simply explained away as being related to their pegged or floating nature.

In order to explore whether the nature of the fluctuation distribution of a particular currency could be related to the characteristics of the underlying economy, the countries to which these currencies belong are grouped into three categories, viz., developed, emerging and frontier markets, as per the Morgan Stanley Capital International (MSCI) market classification framework [26]. This is done on the basis of several criteria such as, the sustainability of economic development, number of companies meeting certain size and liquidity criteria, ease of capital flow, as well as, efficiency and stability of the institutional framework.

To make more explicit the connection between deviation from universality and the heterogeneity of the constituents of the FOREX market, we have examined in detail certain macro-economic factors characterizing a national economy for the role they may play in determining the nature of the fluctuation dynamics of a currency. In particular, we find that a prominent role is played by (a) the GDP per capita $g$, as well as, (b) the Theil index $T$ of export products, which we define below.

The GDP per capita of a country is obtained by dividing the annual economic output, i.e., the aggregate value of all final goods and services produced in it during a year, by the total population. It is one of the primary indicators of the economic performance of a country, with higher GDP per capita indicating a higher standard of living for the people living in it [27]. The annual GDP per capita of the countries whose currencies have been included in our study are obtained from publicly accessible data available in the website of the International Monetary Fund (IMF) 28]. We have averaged the data over 
TABLE I: The currencies of developed (1-14), emerging (15-44) and frontier (45-75) economies considered in the study. The columns indicate the currency code along with the nature of the exchange rate regime (as obtained from Oanda and XE sites), the character of the economy (as categorized by MSCI), the geographical region, the average GDP per capita (provided by IMF) and the mean Theil index (calculated from data available from MIT OEC) for the corresponding countries.

\begin{tabular}{|c|c|c|c|c|c|c|c|}
\hline Sl. no. & Currency & Code & $\begin{array}{c}\text { Exchange Rate Regime } \\
\text { (Oanda, XE) }\end{array}$ & $\begin{array}{l}\text { Market Type } \\
(\text { MSCI })\end{array}$ & Region & $\begin{array}{c}\langle g\rangle \text { in USD } \\
(\mathrm{IMF})\end{array}$ & $\begin{array}{c}\langle T\rangle \\
(\mathrm{MIT}) \\
\end{array}$ \\
\hline 1 & Canadian Dollar & CAD & Floating & Developed & Americas & 32561.46 & 1.95 \\
\hline 2 & Danish Krone & DKK & Pegged within horizontal band & Developed & Europe & 44617.1 & 1.49 \\
\hline 3 & Euro & EUR & Floating & Developed & Europe & 28200.99 & - \\
\hline 4 & Great Britain Pound & GBP & Floating & Developed & Europe & 32126.2 & 1.54 \\
\hline 5 & Iceland Krona & ISK & Floating & Developed & Europe & 39213.54 & 3.69 \\
\hline 6 & Norwegian Kroner & NOK & Floating & Developed & Europe & 59286.29 & 3.45 \\
\hline 7 & Swedish Krona & SEK & Floating & Developed & Europe & 39571.51 & 1.63 \\
\hline 8 & Swiss Franc & $\mathrm{CHF}$ & Floating & Developed & Europe & 52059.39 & 1.96 \\
\hline 9 & Israeli New Shekel & ILS & Floating & Developed & Middle East & 22478.26 & 2.64 \\
\hline 10 & Australian Dollar & AUD & Floating & Developed & Asia-Pacific & 35251.16 & 2.38 \\
\hline 11 & Hong Kong Dollar & HKD & Fixed peg & Developed & Asia-Pacific & 27406.74 & 1.98 \\
\hline 12 & Japanese Yen & JPY & Floating & Developed & Asia-Pacific & 36942.47 & 1.95 \\
\hline 13 & New Zealand Dollar & NZD & Floating & Developed & Asia-Pacific & 23459.35 & 2.14 \\
\hline 14 & Singapore Dollar & SGD & Floating & Developed & Asia-Pacific & 30538.39 & 2.65 \\
\hline 15 & Bolivian Boliviano & $\mathrm{BOB}$ & Crawling peg & Emerging & Americas & 1287.16 & 3.65 \\
\hline 16 & Brazilian Real & BRL & Floating & Emerging & Americas & 6254.18 & 1.93 \\
\hline 17 & Chilean Peso & CLP & Floating & Emerging & Americas & 7563.51 & 3.23 \\
\hline 18 & Colombian Peso & COP & Floating & Emerging & Americas & 3864.52 & 3.01 \\
\hline 19 & Dominican Republic Peso & DOP & Floating & Emerging & Americas & 3509.27 & 2.84 \\
\hline 20 & Mexican Peso & MXN & Floating & Emerging & Americas & 7556.32 & 2.15 \\
\hline 21 & Peruvian Nuevo Sol & PEN & Floating & Emerging & Americas & 3243.22 & 2.99 \\
\hline 22 & Venezuelan Bolivar & VEB & Fixed peg & Emerging & Americas & 6302.1 & 4.85 \\
\hline 23 & Albanian Lek & ALL & Floating & Emerging & Europe & 2319.21 & 2.77 \\
\hline 24 & Czech Koruna & $\mathrm{CZK}$ & Floating & Emerging & Europe & 11701.17 & 1.44 \\
\hline 25 & Hungarian Forint & HUF & Pegged within horizontal band & Emerging & Europe & 9151.13 & 1.87 \\
\hline 26 & Polish Zloty & PLN & Floating & Emerging & Europe & 7866.73 & 1.41 \\
\hline 27 & Russian Rouble & RUB & Floating & Emerging & Europe & 5791.06 & 3.23 \\
\hline 28 & Turkish Lira & TRY & Floating & Emerging & Europe & 6451.81 & 1.58 \\
\hline 29 & Algerian Dinar & DZD & Floating & Emerging & Africa & 2890.28 & 5.17 \\
\hline 30 & Cape Verde Escudo & $\mathrm{CVE}$ & Fixed peg & Emerging & Africa & 2130.76 & 3.71 \\
\hline 31 & Egyptian Pound & EGP & Floating & Emerging & Africa & 1727.67 & 2.73 \\
\hline 32 & Ethiopian Birr & ETB & Floating & Emerging & Africa & 208.91 & 4.33 \\
\hline 33 & Mauritius Rupee & MUR & Floating & Emerging & Africa & 5432.83 & 3.39 \\
\hline 34 & Moroccan Dirham & MAD & Fixed peg & Emerging & Africa & 1997.64 & 2.54 \\
\hline 35 & South African Rand & ZAR & Floating & Emerging & Africa & 4751.66 & 2.14 \\
\hline 36 & Tanzanian Shilling & TZS & Floating & Emerging & Africa & 361.18 & 3.17 \\
\hline 37 & Chinese Yuan Renminbi & CNY & Fixed peg & Emerging & Asia & 2173.96 & 1.55 \\
\hline 38 & Indian Rupee & INR & Floating & Emerging & Asia & 774.57 & 1.74 \\
\hline 39 & Indonesian Rupiah & IDR & Floating & Emerging & Asia & 1630.89 & 1.99 \\
\hline 40 & Papua New Guinea Kina & PGK & Floating & Emerging & Asia & 1014.91 & 4.34 \\
\hline 41 & Philippine Peso & $\mathrm{PHP}$ & Floating & Emerging & Asia & 1440.56 & 3.05 \\
\hline 42 & South Korean Won & KRW & Floating & Emerging & Asia & 15655 & 2.11 \\
\hline 43 & Taiwan Dollar & TWD & Floating & Emerging & Asia & 15707.7 & - \\
\hline 44 & Thai Baht & ТНВ & Floating & Emerging & Asia & 3194.12 & 1.78 \\
\hline 45 & Guatemalan Quetzal & GTQ & Floating & Frontier & Americas & 2134.53 & 2.54 \\
\hline 46 & Honduran Lempira & HNL & Crawling peg & Frontier & Americas & 1380.32 & 3.23 \\
\hline 47 & Jamaican Dollar & JMD & Floating & Frontier & Americas & 4042.29 & 4.25 \\
\hline 48 & Paraguay Guarani & PYG & Floating & Frontier & Americas & 1892.51 & 3.81 \\
\hline 49 & Trinidad Tobago Dollar & TTD & Floating & Frontier & Americas & 12983.73 & 4.21 \\
\hline 50 & Croatian Kuna & HRK & Floating & Frontier & Europe & 9166.72 & 1.75 \\
\hline 51 & Kazakhstan Tenge & $\mathrm{KZT}$ & Floating & Frontier & Europe & 4399.18 & 3.97 \\
\hline 52 & Latvian Lats & LVL & Fixed peg & Frontier & Europe & 6912.26 & 2.35 \\
\hline 53 & Botswana Pula & BWP & Crawling peg & Frontier & Africa & 5447.23 & 5.45 \\
\hline 54 & Comoros Franc & $\mathrm{KMF}$ & Fixed peg & Frontier & Africa & 609.64 & 5.05 \\
\hline 55 & Gambian Dalasi & GMD & Floating & Frontier & Africa & 513.84 & 4.27 \\
\hline 56 & Ghanaian Cedi & GHC & Floating & Frontier & Africa & 871.99 & 4.11 \\
\hline 57 & Guinea Franc & GNF & Fixed peg & Frontier & Africa & 419.31 & 5.03 \\
\hline 58 & Kenyan Shilling & KES & Floating & Frontier & Africa & 586 & 2.95 \\
\hline 59 & Malawi Kwacha & MWK & Floating & Frontier & Africa & 226.69 & 4.5 \\
\hline 60 & Mauritanian Ouguiya & $\mathrm{MRO}$ & Floating & Frontier & Africa & 752.88 & 4.96 \\
\hline 61 & Mozambique Metical & MZM & Floating & Frontier & Africa & 330.54 & 4.28 \\
\hline 62 & Nigerian Naira & NGN & Floating & Frontier & Africa & 782.49 & 6.02 \\
\hline 63 & Sao Tome and Principe Dobra & STD & Fixed peg & Frontier & Africa & 864.42 & 4.15 \\
\hline 64 & Zambian Kwacha & ZMK & Floating & Frontier & Africa & 677.53 & 4.67 \\
\hline 65 & Jordanian Dinar & JOD & Fixed peg & Frontier & Middle East & 2617.85 & 3 \\
\hline 66 & Kuwaiti Dinar & KWD & Fixed peg & Frontier & Middle East & 25554.56 & 5.49 \\
\hline 67 & Syrian Pound & SYP & Fixed peg & Frontier & Middle East & 1732.47 & 4.21 \\
\hline 68 & Brunei Dollar & BND & Fixed peg & Frontier & Asia & 23516.1 & 5.45 \\
\hline 69 & Bangladeshi Taka & $\mathrm{BDT}$ & Floating & Frontier & Asia & 436.09 & 3.63 \\
\hline 70 & Cambodian Riel & KHR & Floating & Frontier & Asia & 498.85 & 3.85 \\
\hline 71 & Fiji Dollar & FJD & Fixed peg & Frontier & Asia & 3052.58 & 3.37 \\
\hline 72 & Lao Kip & LAK & Floating & Frontier & Asia & 577.82 & 3.66 \\
\hline 73 & Pakistan Rupee & PKR & Floating & Frontier & Asia & 756.7 & 2.87 \\
\hline 74 & Samoan Tala & WST & Fixed peg & Frontier & Asia & 2215.73 & 4.63 \\
\hline 75 & Sri Lankan Rupee & LKR & Floating & Frontier & Asia & 1438.09 & 2.65 \\
\hline
\end{tabular}



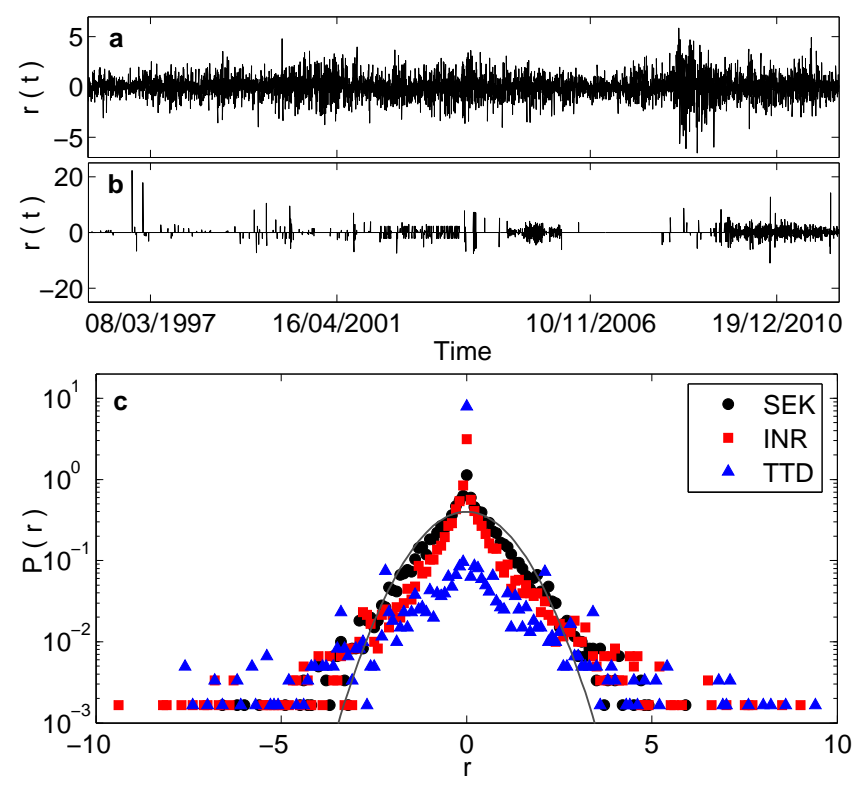

FIG. 1: (color online). Heavy tailed behavior in the distribution of currency exchange rate fluctuations. The time-series of normalized log returns $r(t)$ for currencies of developed economies, e.g., SEK (a), shows relatively lower amplitude variations compared to that of currencies of frontier economies, e.g., TTD (b), in general (note the different scales in the ordinate of the two panels). However, the probability density functions of $r$ for all currencies show a heavy-tailed nature, shown in (c) for currencies from a developed economy, SEK (black, circles), an emerging economy, INR (red, squares), and a frontier economy, TTD (blue, triangles). For comparison, the standard normal distribution is shown using a solid curve.

the 18 year period (1995-2012) considered in our study to obtain the mean GDP per capita $\langle g\rangle$.

The Theil index measures the diversity of the export products of a country [29] and is defined as $T=$ $\frac{1}{M} \sum_{i=1}^{M}\left(\frac{x_{i}}{\bar{x}} \ln \frac{x_{i}}{\bar{x}}\right)$, where $x_{i}$ is the total value (in USD) of the $i$-th export product of a country, $\bar{x}$ is the average value of all export products and $M$ is the total number of different products that are exported. A high value of $T$ corresponds to large heterogeneity in the values of the different exported products, indicating that a few products dominate the export trade. By contrast, low $T$ implies that a country has a highly diversified portfolio of export products and therefore, relatively protected from the vagaries of fluctuations in the demand for any single product. To compute the Theil index we have used the annual export product data of different countries available from the Observatory of Economic Complexity (OEC) at MIT [30]. We have used the four digit level of the Standard International Trade Classification for categorizing different products which corresponds to $M=777$ distinct export products in the data set. We have averaged the annual Theil indices over the period 1995-2012 to obtain the mean Theil index $\langle T\rangle$ for each country.
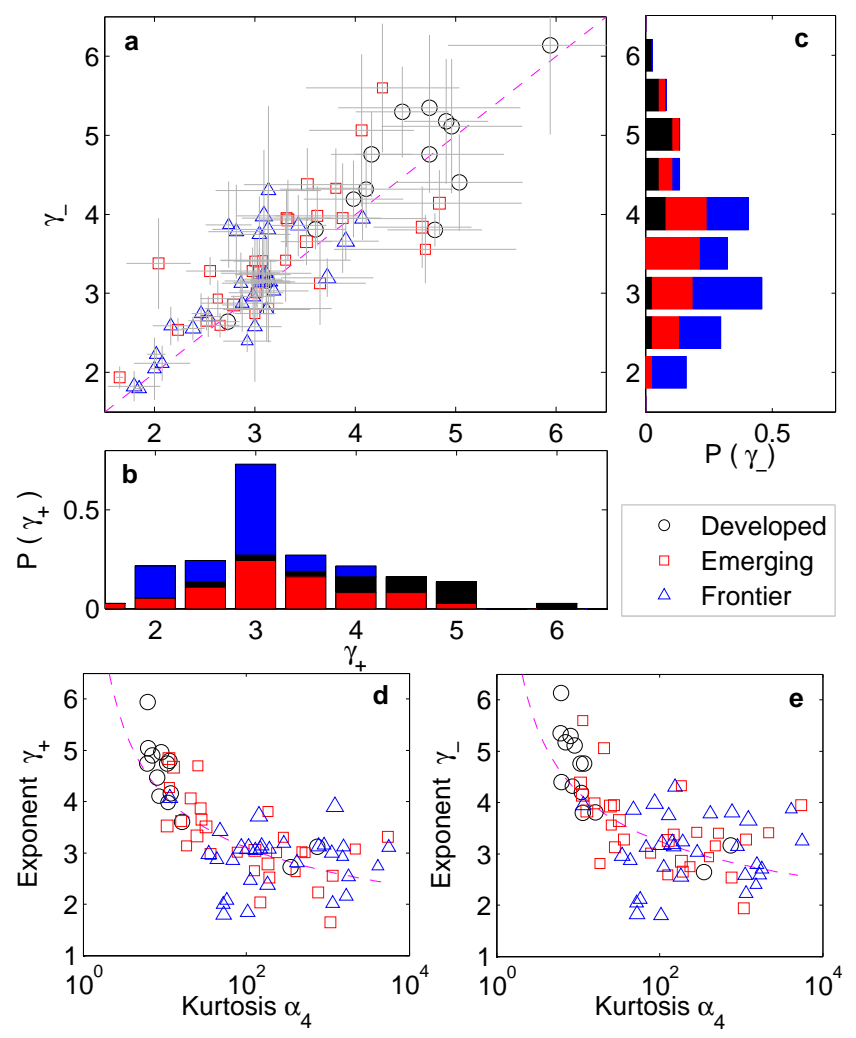

FIG. 2: (color online). (a-c) Deviation from universality for exchange rate fluctuations. The probability distribution of the power law exponents $\gamma_{+}$(b) and $\gamma_{-}$(c) obtained by maximum likelihood estimation (MLE) for the positive and negative tails, respectively, of the individual return distributions for the 75 currencies, show a peak around 3 with median values of 3.11 (for $\gamma_{+}$) and 3.28 (for $\gamma_{-}$). Error bars indicate the uncertainty in the estimated values and are obtained by a non-parametric bootstrap technique. Points lying closer to the diagonal $\left(\gamma_{+}=\gamma_{-}\right.$, indicated by a broken line) in (a) imply a higher degree of symmetry in the distribution of $r$ for the corresponding currency, i.e., positive and negative fluctuations of similar magnitude are equally probable. The heavy-tailed nature of the distributions characterized by the tail-exponents correspond closely to their peakedness measured using the kurtosis $\alpha_{4}$, as shown by the scatter plot between (d) $\alpha_{4}$ and $\gamma_{+}$and (e) $\alpha_{4}$ and $\gamma_{-}$for the currencies. The best log-linear fits, indicated by broken lines, correspond to $\alpha_{4}=\exp \left[\left(\gamma_{ \pm} / A_{ \pm}\right)^{-\beta_{ \pm}}\right]$with $A_{+}=5.8, \beta_{+}=2.4$ (d) and $A_{-}=5.6, \beta_{-}=2.8(\mathrm{e})$. The Pearson correlation coefficient between $\log \left(\log \left(\alpha_{4}\right)\right)$ and $\log \left(\gamma_{ \pm}\right)$are $\rho=-0.67$ $\left(p=10^{-11}\right)$ for $(\mathrm{d})$ and $\rho=-0.59\left(p=10^{-8}\right)$ for (e). Different symbols and colors are used to indicate currencies from developed (black, circles), emerging (red, squares) and frontier (blue, triangles) economies, while symbol size is proportional to $\log (\langle g\rangle)$ of the corresponding countries.

\section{RESULTS}

We have measured the fluctuations in the exchange rates of 75 currencies (see data description for details) 
with respect to the US Dollar over the period 1995-2012. To ensure that the result is independent of the unit of measurement, we have quantified the variation in the exchange rate $P_{i}(t)$ of the $i$-th currency $(i=1, \ldots, N)$ at time $t$ by its logarithmic return defined over a timeinterval $\Delta t$ as $R_{i}(t, \Delta t)=\ln P_{i}(t+\Delta t)-\ln P_{i}(t)$. As explained in the data description, our data comprises daily exchange rates and we therefore consider $\Delta t=1$ day. Different currencies can vary in terms of the intensity of fluctuations in their exchange rates (volatility) as can be measured by the standard deviation $\sigma$ of the returns. Thus, to compare the return distributions of the different currencies, we normalize the returns of each currency $i$ by subtracting the mean value $\left\langle R_{i}\right\rangle=$ $\Sigma_{t=1}^{\tau-1} R_{i}(t) /(\tau-1)$ and dividing by the standard deviation $\sigma_{i}(t)=\sqrt{\frac{1}{\tau-2} \Sigma_{t^{\prime} \neq t}\left[R_{i}\left(t^{\prime}\right)-\left\langle R_{i}\right\rangle\right]^{2}}$ (removing the self contribution from the measure of volatility), obtaining the normalized return, $r_{i}(t)=\left(R_{i}(t)-\left\langle R_{i}\right\rangle\right) / \sigma_{i}(t)$.

\section{A. The "inverse square law" of the distribution of fluctuations for currency exchange rates}

As can be seen from Fig. 10(a-b), the returns quantifying the fluctuations in the exchange rate of currencies can appear extremely different even though they have been normalized by their volatilities. The temporal variation of $r(t)$ for SEK [shown in Fig. 1 (a)], the currency of a developed economy, is mostly bounded between a narrow interval around 0 with the fluctuations never exceeding 6 standard deviations from the mean value. By contrast, Fig. 1 (b) shows that TTD, belonging to a frontier economy, frequently exhibits extremely large fluctuations that can occasionally exceed even 20 standard deviations - an event extremely unlikely to have been observed had the distribution been of a Gaussian nature [31]. These observations suggest that the distributions of the exchange rate fluctuations have long tails and that different currencies may have significantly different nature of heavytailed behavior. As shown in Fig. 1 (c), where the distributions of $r$ for SEK, TTD and an emerging economy currency, INR, is displayed, this is indeed the case.

The nature of the tails of the return distributions is established quantitatively by fitting them to a power-law decay for the probability distribution having the functional form $P(r) \sim r^{-\gamma}$ through maximum likelihood estimation (MLE) [32]. Uncertainty in estimating the optimal value of $\gamma$ is calculated by performing MLE of exponents from 100 surrogate data-sets for each currency. These are constructed by random sampling with replacement from the original return time-series data [32]. While both the positive and negative returns show heavy tails, we note that the exponents characterizing them need not be identical for a currency, such that the corresponding return distribution is asymmetric or skewed. The scatter plot in Fig. 2 (a) shows how the positive and negative tail exponents, $\gamma_{+}$and $\gamma_{-}$respectively, are related to each other for the different currencies. Currencies that occur closer to the diagonal line $\gamma_{+}=\gamma_{-}$have similar nature of upward and downward exchange rate movements. However, currencies which occur much above the diagonal (i.e., $\gamma_{+}<\gamma_{-}$) will tend to have a higher probability of extreme positive returns compared to negative ones, while those below the diagonal are more likely to exhibit very large negative returns. We note in passing that the skewness depends, to some extent, on the state of the economy of the country to which a currency belongs, with return distributions of developed economies being the least asymmetric in general, having mean skewness $0.52 \pm 1.28$, while those of emerging and frontier economies are relatively much higher, being $6.54 \pm 15.24$ and $6.60 \pm 18.04$, respectively.

The distribution of the exponents characterizing the power-law nature of the exchange-rate returns shown in Figs. 2(b-c) peaks around 3 for both the positive and negative tails. As a probability distribution function with a power law characterized by exponent value $\gamma \simeq 3$ implies that the corresponding CCDF also has a power-law form but with exponent value $\alpha=\gamma-1 \simeq 2$ [33], this result suggests an "inverse square law" governing the nature of fluctuations in the currency market in contrast to the "inverse cubic law" that has been proposed as governing the price and index fluctuations in several financial markets [8 -13]. However, as is the case here, such a "law" is only manifested on the average, as the return distributions for individual assets can have quite distinct exponents [11]. Here, we observe that the different currencies can have exponents as low as 2 and as high as 6. Moreover, there appears to be a strong correlation between the nature of the tail and the state of the underlying economy to which the currency belongs. Thus, developed economy currencies tend to have the largest exponents, while most of the lowest values of exponents belong to currencies from the frontier economies. This suggests an intriguing relation between the nature of currency fluctuations and the state of the underlying economy, that could possibly be quantified by one or more macroeconomic indicators. This theme is explored in detail below.

In order to verify that the nature of fluctuations in exchange rates does not change drastically depending on the specific choice of base currency, we have re-calculated the exponents $\gamma$ characterizing return distributions of different currencies that are obtained using each of the 75 currencies as the base. Fig. 3] shows that for all base currencies used in our study, the exponents $\gamma_{+}$ are distributed about mean values that fluctuate around $\left\langle\left\langle\gamma_{+}\right\rangle\right\rangle \sim 3$ (a similar behavior is seen for the exponents of the negative returns distributions, $\gamma_{-}$). This suggests that the inverse square law form for the heavy tails of return distributions is valid on average relatively independent of the base currency used to calculate the exchange rates.

The character of the heavy tails of the returns $r$ is closely related to the peaked nature of the distribution 

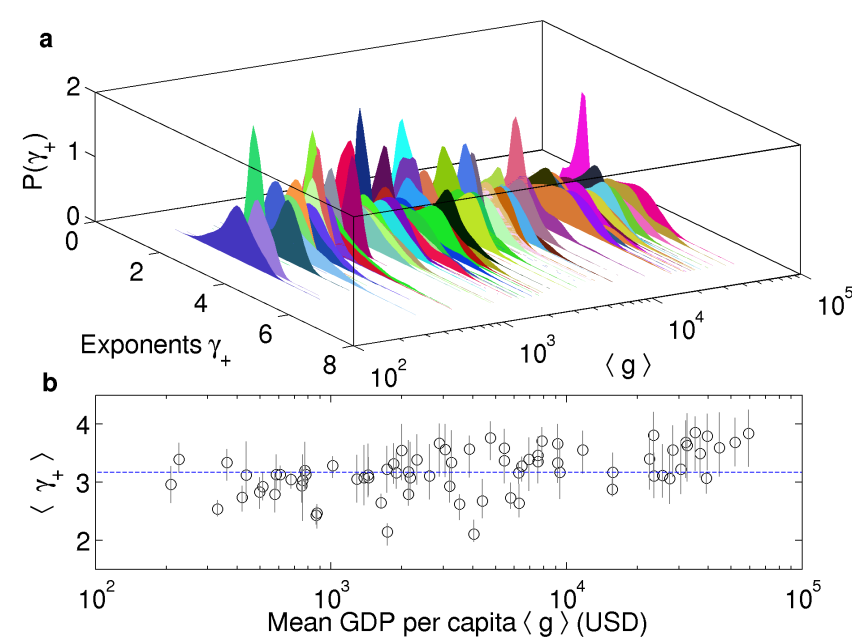

FIG. 3: (color online). Distribution of fluctuations for currency exchange rates with respect to different base currencies display an "inverse square law" on average. (a) The ensemble of distributions of power-law exponents $\gamma_{+}$ for the positive return distributions of 75 currencies calculated with respect to each of 75 base currencies that are arranged according to the mean GDP per capita $\langle g\rangle$ of the corresponding economy (of the base currency). (b) The mean values of the exponents $\gamma_{+}$(circles) obtained using each of the base currencies are almost all clustered around the value of 3 , indicating an "inverse square law" behavior of the heavy tails of return distributions that is relatively stable against the choice of different bases for measuring the fluctuation. Error bars indicated represent the standard deviation in the estimated values of $\gamma_{+}$for different currencies for a given base currency. The broken line represents the grand average $\left(\left\langle\left\langle\gamma_{+}\right\rangle\right\rangle=3.21\right)$ of the values for the the exponent $\gamma_{+}$, taken over all currencies and bases.

that can be quantified by its kurtosis which is defined as $\alpha_{4}=E(r-\mu)^{4} / \sigma^{4}$, where $E()$ is the expectation while $\mu$ and $\sigma$ are the mean and standard deviation, respectively, of $r$. Fig. 2 (d-e) shows the relation between the kurtosis and the exponents for the tails of the return distributions of the different currencies. The fitted curve shown qualitatively follows the theoretical relation between the two which can be derived by assuming that the distribution is Pareto, i.e., follows a power law (although for such a situation, the kurtosis is finite only for exponent values $\gamma>5$ ). We observe that the relation between the exponents and kurtosis suggested by the scatter plots can be approximately fit by the function $\alpha_{4} \sim \exp \left[\left(\gamma_{ \pm} / A_{ \pm}\right)^{-\beta_{ \pm}}\right]$with $\beta_{+}=2.4, A_{+}=5.8$ for the positive tail and $\beta_{-}=2.8, A_{-}=5.6$ for the negative tail [Fig. 2 (d) and (e),respectively]. The strong correlation between the peakedness of the distribution and the character of the heavy tails can be quantified by the Pearson correlation coefficients between $\log \left(\gamma_{ \pm}\right)$and $\log \left(\log \left(\alpha_{4}\right)\right)$, viz., $\rho=-0.67\left(p=10^{-11}\right)$ for the positive returns and $\rho=-0.59\left(p=10^{-8}\right)$ for the negative returns. Thus, instead of using two different exponent values (corresponding to the positive and negative tails) for each return distribution, we shall henceforth focus on the single kurtosis value that characterizes the distribution.

\section{B. Deviation from universality related to macroeconomic factors}

Given the variation in the nature of fluctuation distribution of different currencies from a single universal form, we ask whether the deviations are systematic in nature. Note that, the currencies belong to countries having very diverse economies, that trade in a variety of products \& services with other countries and which may have contrasting economic performances. An intuitive approach would be to relate the differences in the return distributions with metrics which capture important aspects of the economies as a whole. Fig. 4 shows that there is indeed a significant correlation between the kurtosis of the return distributions for the currencies and two macroeconomic indicators of the underlying economies, viz., the mean GDP per capita, $\langle g\rangle$, and the mean Theil index, $\langle T\rangle$, that describe the overall prosperity and the diversity of export products, respectively (see data description for details).

Fig. (4) (a) shows that the scatter of kurtosis $\alpha_{4}$ against $\langle g\rangle$ can be approximately fit by a power law of the form: $\alpha_{4} \sim\langle g\rangle^{-2.2}$. The Pearson correlation coefficient between the logarithms of the two quantities is $\rho=-0.55$ $\left(p=10^{-7}\right)$. Thus, in general, currencies of countries having higher GDP per capita tend to be more stable, in the sense of having low probability of extremely large fluctuations. However, there are exceptions where currencies exhibit high kurtosis even when they belong to countries with high GDP per capita (e.g., HKD and ISK which are indicated in the figure). In these cases, the peakedness of the distribution may reflect underlying economic crises, e.g., the 2008 Icelandic financial crisis in the case of ISK and the 2003 SARS crisis for HKD. Furthermore, we observe that currencies belonging to high GDP per capita economies that are dependent on international trade of a few key resources - such as, crude oil - also exhibit high kurtosis (e.g., KWD and BND). This suggests a dependence of the nature of the fluctuation distribution on the diversity of their exports, which is indeed shown in Fig. 4 (b). The dependence of the kurtosis on $T$ (which is a measure of the variegated nature of trade) of the corresponding economy is approximately described by a power-law relation: $\alpha_{4} \sim\langle T\rangle^{9.1}$. The Pearson correlation coefficient between the logarithms of the two quantities is $\rho=0.53\left(p=10^{-6}\right)$. This implies that, in general, currencies of countries having low $\langle T\rangle$, i.e., having welldiversified export profile, tend to be more stable.

Note that the fluctuations of the currencies depend on both of these above macroeconomic factors, and the differences in their nature cannot be explained exclusively by any one of them. It is therefore meaningful to per- 
form a multi-linear regression of $\alpha_{4}$ as a function of both GDP per capita and Theil index using an equation of the form: $\log \left(\alpha_{4}\right)=b_{0}+b_{1} \log (\langle g\rangle)+b_{2} \log (\langle T\rangle)$, where the constants $b_{0}(=6.74), b_{1}(=-0.48)$ and $b_{2}(=1.69)$ are the best-fit regression coefficients. The coefficient of determination $R^{2}$, which measures how well the data fits the statistical model, is found to be $0.39\left(p \simeq 10^{-8}\right)$. This indicates that together the macroeconomic factors of GDP per capita (related to the overall economic performance) and Theil index (related to the international trade of the country) explain over $39 \%$ of the variation between the nature of the return distributions of the different currencies.

One of the assumptions of multi-linear regression analysis is that the explanatory variables [viz., $\log (\langle g\rangle)$ and $\log (\langle T\rangle)]$ are not highly correlated with each other. Thus we need to explicitly test for the absence of significant collinearity, i.e., linear dependence of one explanatory variable on the other variables. A commonly used indicator of collinearity is the variance inflation factor $(V I F)[34]$. When the variation of a specific explanatory variable (referred to as a predictor) is largely explained by a linear combination of the other predictors, VIF for that predictor is correspondingly large. Complete absence of collinearity corresponds to the case $V I F=1$ and the inflation is measured relative to this reference value. VIF have been shown to correspond to the diagonal elements of the inverse of the matrix of correlations between the predictors [34] and using this method we obtain $V I F=1.28$ for both the macroeconomic factors considered by us. As commonly collinearity is considered to be a cause for concern only if VIF values are higher than 5, GDP per capita and Theil index can be reasonably treated as independent explanatory variables in our analysis. We have also investigated the possible dependence of the nature of the fluctuation distribution on other economic factors, such as the foreign direct investment (FDI) net inflow, but none of these appear to be independent of the two factors considered above.

To investigate the reason for the strong relation between the kurtosis of the return distribution for a currency and the corresponding underlying macroeconomic factors, we need to delve deeper into the nature of the dynamics of the exchange rate fluctuations. For this we first look into the self-similar scaling behavior of the timeseries of exchange rate of a currency $P(t)$ using the detrended fluctuation analysis (DFA) technique suitable for analyzing non-stationary processes with long-range memory 35]. Here, a time-series is de-trended over different temporal windows of sizes $s$ using least-square fitting with a linear function. The residual fluctuations $F(s)$ of the resulting sequence, measured in terms of the standard deviation, is seen to scale as $F(s) \sim s^{\gamma_{D F A}}$, where $\gamma_{D F A}$ is referred to as the DFA exponent. The numerical value of this exponent (lying between 0 and 1) provides information about the nature of the fractional Brownian motion undertaken by the system. For $\gamma_{D F A} \simeq 1 / 2$, the process is said to be equivalent to a random walk subject

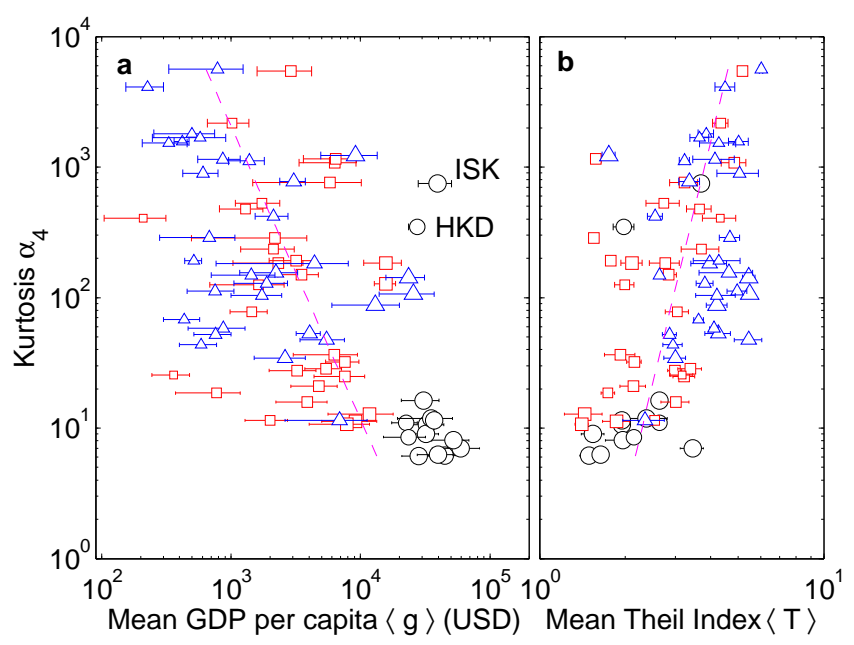

FIG. 4: (color online). Variation of the kurtosis $\alpha_{4}$ of exchange rate fluctuation distributions of different currencies with (a) annual GDP per capita, $\langle g\rangle$ (in USD) and (b) annual Theil index of the export products, $\langle T\rangle$, for the corresponding countries, averaged over the period 1995-2012. The Pearson correlation coefficient between $\log (\langle g\rangle)$ and $\log \left(\alpha_{4}\right)$ is $\rho=-0.55$ $\left(p=10^{-7}\right)$, the best-fit functional relation between the two being $\alpha_{4} \sim\langle g\rangle^{-2.2}$. Currencies of developed economies that are outliers from this general trend, viz., ISK and HKD that have high kurtosis despite having high GDP per capita, are explicitly indicated in (a). A similar analysis shows that the Pearson correlation coefficient between $\log (\langle T\rangle)$ and $\log \left(\alpha_{4}\right)$ is $\rho=0.53\left(p=10^{-6}\right)$, with the best-fit functional relation being $\alpha_{4} \sim\langle T\rangle^{9.1}$. Different symbols are used to indicate currencies from developed (black, circles), emerging (red, squares) and frontier (blue, triangles) economies, while symbol size is proportional to $\log (\langle g\rangle)$ of the corresponding countries. Error bars represent the standard deviation of the annual values of $g$ and $T$ over the period 1995-2012 for the countries corresponding to each currency.

to white noise, while $\gamma_{D F A}>1 / 2(<1 / 2)$ implies that the time-series is correlated (anti-correlated). As seen from Fig. [5] (a), the DFA exponents of currencies for most developed economies - which also have the lowest kurtosis - are close to 0.5, indicating that these currencies are following uncorrelated random walk [36]. In contrast, currencies of the emerging and frontier economies, possessing higher values of kurtosis, typically have $\gamma_{D F A}<0.5$ indicating sub-diffusive dynamics.

To understand the reason for this sub-diffusive behavior we have analyzed the exchange rate time-series using the variance ratio (VR) test. This technique, based on the ratio of variance estimates for the returns calculated using different temporal lags, is often used to find how close a given time-series is to a random walk [37]. For a sequence of $\log$ returns $\left\{R_{t}\right\}$, the variance ratio for a lag $l$ is defined as:

$$
V R(l)=\frac{\sum_{k=l}^{\tau}\left(\sum_{t=k-l}^{k-1} R_{t}-l \mu_{R}\right)^{2}}{\sigma_{R}^{2} l(\tau-l+1)(1-[l / \tau])},
$$




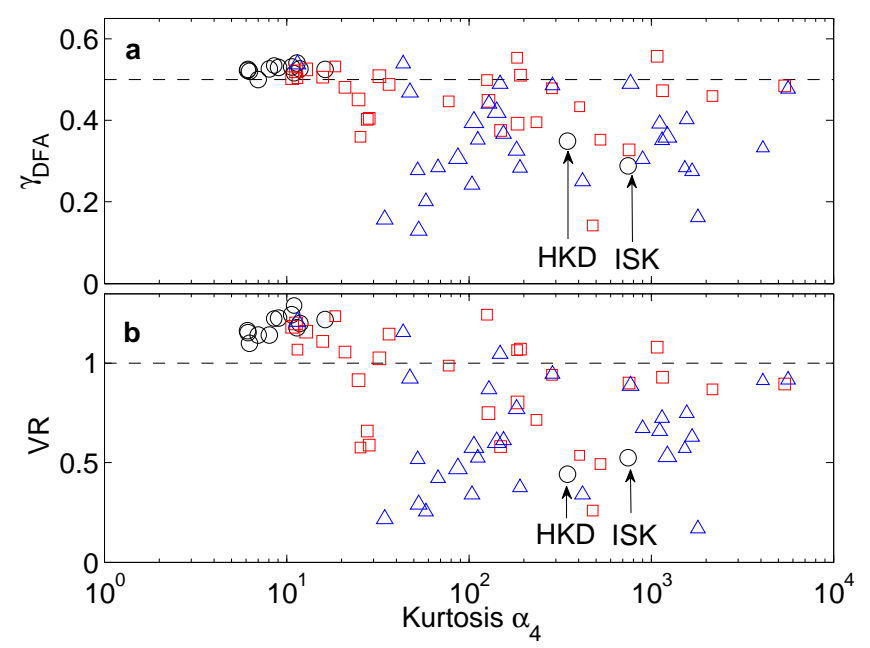

FIG. 5: (color online). Variation of (a) the long-range auto-correlation scaling exponent $\gamma_{D F A}$ obtained using detrended fluctuation analysis of the exchange rate time series, and (b) the variance ratio $(V R)$ of the exchange rate fluctuations calculated using lag $l$ (= 10 days), with the kurtosis $\alpha_{4}$ of the normalized logarithmic return distributions of different currencies. Different symbols are used to indicate currencies from developed (black, circles), emerging (red, squares) and frontier (blue, triangles) economies, while symbol size is proportional to $\log (\langle g\rangle)$ of the corresponding countries. The broken lines in (a) and (b) indicate the values of $\gamma_{D F A}(=0.5)$ and $V R(=1)$ corresponding to an uncorrelated random walk. Currencies of developed economies that are outliers, viz., ISK and HKD that have much higher kurtosis than others in the group, are explicitly indicated.

where $\mu_{R}=\left\langle R_{t}\right\rangle$ and $\sigma_{R}^{2}=\left\langle\left(R_{t}-\mu_{R}\right)^{2}\right\rangle$ are the mean and variance of the $\left\{R_{t}\right\}$ sequence. An uncorrelated random walk is characterized by a VR value close to 1 . If $V R>1$, it indicates mean aversion in the time-series, i.e., the variable has a tendency to follow a trend where successive changes are in the same direction. In contrast, $V R<1$ suggests a mean-reverting series where changes in a given direction are likely to be followed by changes in the opposite direction preventing the system from mov-

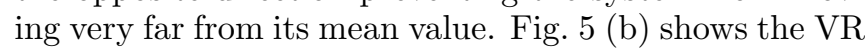
values for different currencies, calculated using lag $l=10$ days, as a function of their kurtosis. Consistent with the DFA results reported above, it is seen that for currencies of developed economies the VR is close to 1, indicating uncorrelated Brownian diffusion as the nature of their exchange rate dynamics. However, for most frontier and a few emerging economy currencies, the VR value is substantially smaller than 1 , implying that their trajectories have a mean-reverting nature. As in Fig. 4, we note that HKD and ISK appear as outliers in Fig. 5 in that, although belonging to the group of countries having high GDP per capita, they share the characteristics shown by most emerging and frontier economies.

We can now understand the sub-diffusive nature of the dynamics of these currencies as arising from the anticorrelated nature of their successive fluctuations which prevents excursions far from the average value. Thus, when we consider the time-series of all currencies after normalizing their variance, the fluctuations of the emerging and frontier economy currencies mostly remain in the neighborhood of the average value with rare, occasional deviations that are very large compared to developed economy currencies. This accounts for the much heavier tails of the return distributions of the former and the corresponding high value of kurtosis. It is intriguing to consider whether the difference in the nature of the movement of exchange rates of the currencies could be possibly related to the role played by speculation in the trading of these currencies [38]. We also note that these results are in broad agreement with the fact that efficient markets follow uncorrelated random walks and the notion that the markets of developed economies are far more efficient than those of emerging and frontier ones. A temporally resolved analysis of the nature of the distributions at different periods shows strong disruption of the otherwise regular pattern of systematic deviation during the severe crisis of 2008-09, indicating its deeprooted nature affecting the real economy.

\section{Temporal evolution of system properties}

In the analysis presented above we have considered the entire temporal duration which our data-set spans. However, as the world economy underwent significant changes during this period, most notably, the global financial crisis of 2008 , it is of interest to see how the properties we investigate have evolved with time. For this purpose we divide the data-set into three equal non-overlapping periods each comprising 2011 days, corresponding to Period I: Oct 23, 1995 - Apr 25, 2001, Period II: Apr 26, 2001 - Oct 28, 2006 and Period III: Oct 29, 2006 - Apr 30, 2012. Note that the last period corresponds to the crisis of the global economy spanning 2007-2009. For each of these, we carry out the same procedures as described earlier in the context of the the entire data-set. As seen from Fig. 6. the behavior in the first two intervals appear to be quite similar in terms of the various properties that have been measured, but large deviations are seen in the third interval. This is apparent both for the relation between kurtosis and mean GDP per capita [Fig. 6 (a-c)], as well as that between kurtosis and mean Theil index (figure not shown). The dependence of the nature of the fluctuation distribution on the properties of the underlying economy seem to have weakened in Period III. For example, while there is significant strong negative correlation between $\log (\langle g\rangle)$ and $\log \left(\alpha_{4}\right)$ for the first two intervals, viz., $\rho=-0.60\left(p=10^{-8}\right)$ and -0.57 $\left(p=10^{-8}\right)$, respectively, it decreases to only $\rho=-0.28$ $\left(p=10^{-2}\right)$ for the third interval. Furthermore, the first two intervals show a $1 /\langle g\rangle^{2}$ dependence of the kurtosis $\alpha_{4}$, same as that seen for the entire period that we have 

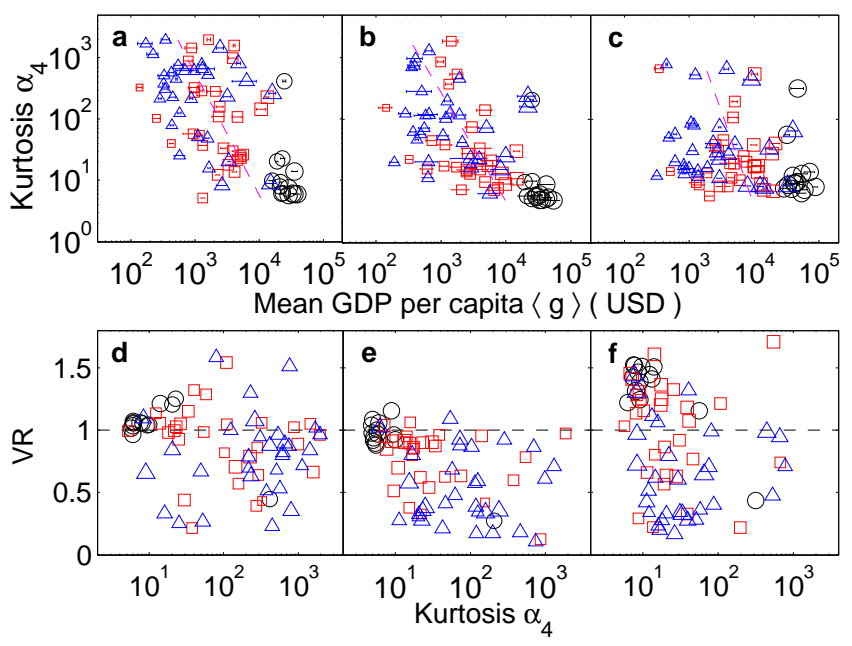

FIG. 6: (color online). Temporal evolution of the statistical properties of exchange rate fluctuation distributions of different currencies. The variation of (a-c) the kurtosis $\alpha_{4}$ of the distributions with annual GDP per capita, $g$ (in USD) and that of (d-f) the variance ratio (VR) of the different normalized fluctuations time series with kurtosis $\alpha_{4}$, are shown for three different periods, viz., Period I: Oct 23, 1995 - Apr 25, 2001 (a \& d), Period II: Apr 26, 2001 - Oct 28, 2006 (b \& e) and Period III: Oct 29, 2006 - Apr 30, 2012 (c $\& \mathrm{f})$, which divide the duration under study into three equal, non-overlapping segments. The GDP per capita of the different countries for each period are obtained by averaging the annual values over the corresponding periods. The Pearson correlation coefficients between $\log (\langle g\rangle)$ and $\log \left(\alpha_{4}\right)$ for the three periods are $\rho_{I}=-0.60\left(p\right.$-value $\left.=10^{-8}\right), \rho_{I I}=-0.57$ $\left(p\right.$-value $\left.=10^{-8}\right)$ and $\rho_{I I I}=-0.28\left(p\right.$-value $\left.=10^{-2}\right)$. For the first two periods, the best-fit functional relation between the two is $\alpha_{4} \sim 1 /\langle g\rangle^{2}$, while for the third period, the dependence of $\alpha_{4}$ on $\langle g\rangle$ shows a strong deviation from the inverse square relation seen in the other two periods. Comparing the variance ratio values for the three different periods show a higher degree of mean aversion in the third period. Period III, during which the major economic crisis of 2008-09 occurred, is distinguished by large deviation from the trends seen in the other two periods. Different symbols are used to indicate currencies from developed (black, circles), emerging (red, squares) and frontier (blue, triangles) economies, while symbol size is proportional to $\log (\langle g\rangle)$ of the corresponding countries.

reported above. However, this is not true for the last interval where the best fit for the dependence of $\alpha_{4}$ on $\langle g\rangle$ shows a strong deviation from the behavior seen in other periods. Similarly, we have found significant high correlation between $\log (\langle T\rangle)$ and $\log \left(\alpha_{4}\right)$, corresponding to Pearson coefficients $\rho=0.50\left(p=10^{-6}\right)$ and $\rho=0.46$ $\left(p=10^{-5}\right)$, respectively, for the first two intervals. In contrast, for the third interval we observe a relatively smaller correlation $\rho=0.35\left(p=10^{-2}\right)$. In addition, the relation between the variance ratio and the kurtosis of the returns [Fig. 6 (d-f)], as well as that between the DFA exponent and the kurtosis (figure not shown), are seen to be similar in the first two intervals but very different in the third - in part because the VR for the developed and some emerging economies have adopted values $>1$ (i.e., exhibiting mean aversion) in this last interval, while earlier they were close to 1 (i.e., similar to a random walk). While Periods I and II had their share of economic booms and busts, it is instructive to note that the 2008 crisis was severe enough to disrupt systemic features that were otherwise maintained over time.

\section{DISCUSSION AND CONCLUSION}

The work we report here underscores the importance of studying economic systems, especially financial markets, for gaining an understanding of the collective dynamics of heterogeneous complex systems. At the largest scale, such a system encompasses the entire world where the relevant entities are the different national economies interacting with each other through international trade and the foreign exchange market. The far-from-equilibrium behavior of this highly heterogeneous complex system has been investigated here by focusing on the fluctuations of exchange rates of the respective currencies. Understanding the overall features of this dynamics is crucially important in view of the human and social cost associated with large-scale disruptions in the system, as was seen during the recent 2008 world-wide economic crisis.

Our results suggest a putative invariant signature in the dynamics of exchange rates, possibly the first such seen in macroeconomic phenomena. This is in contrast to microeconomic systems like individual financial markets where robust stylized facts such as the "inverse cubic law" has been established for some time. The "inverse square law" that we report here also has a fundamental distinction in that distributions characterized by CCDF exponents $\alpha \leq 2$ belong to the Levy-stable regime. By contrast, the logarithmic return distributions of equities and indices of financial markets that have exponent values around 3 are expected to converge to a Gaussian form at longer time scales [13, 39]. It suggests that extreme events corresponding to sudden large changes in exchange rates, in particular for currencies belonging to emerging and frontier economies, should be expected far more often compared to other financial markets. The "inverse square law" has recently been also reported in at least one other market, viz., that of Bitcoins in the initial period following its inception [40]. We note that agent-based modeling of markets suggest that such a distribution can arise if market players are relatively homogeneous in their risk propensity [41, 42].

To conclude, the results of our study help in revealing a hidden pattern indicative of relative invariance in a highly heterogeneous complex system, viz., the FOREX market. The robust empirical feature that we identify here is a power law characterizing the heavy-tailed nature of the fluctuation distributions of exchange rates for different currencies. The systematic deviation of individual currencies from the universal form (the "inverse 
square law"), quantified in terms of their kurtosis measuring the peakedness of the return distributions, can be linked to metrics of the economic performance and degree of diversification of export products of the respective countries. By doing detrended fluctuation analysis, the distinct behavior of currencies corresponding to developed, emerging and frontier markets can be linked to the different scaling behaviors of the random walks undertaken by these currencies. Our work shows how robust empirical regularities among the components of a complex system can be uncovered even when the system is characterized by a large number of heterogeneous interacting elements exhibiting distinct local dynamics. Simi- lar approaches may be used for identifying invariances in other biological and socio-economic systems.

\section{Acknowledgments}

We thank Anindya S. Chakrabarti, Tanmay Mitra and V. Sasidevan for helpful suggestions. We gratefully acknowledge the assistance of Uday Kovur in the preliminary stages of this work. This work was supported in part by IMSc Econophysics (XII Plan) Project funded by the Department of Atomic Energy, Government of India.
[1] K. G. Wilson, Rev. Mod. Phys. 55, 583 (1983).

[2] L. Henrickson and B. McKelvey, Proc. Natl. Acad. Sci. USA 99, 7288 (2002).

[3] H. Hinrichsen, Adv. Phys. 49, 815 (2000).

[4] R. Cohen, D. Ben-Avraham and S. Havlin, Phys. Rev. Lett. 66, 036113 (2002).

[5] Bank for International Settlements, Triennial Central Bank Survey: Global foreign exchange market turnover in 2013 (2014).

[6] Y. Yura, H. Takayasu, D. Sornette, and M. Takayasu, Phys. Rev. Lett. 112, 098703 (2014).

[7] S. Sinha, A. Chatterjee, A. Chakraborti and B. K. Chakrabarti, Econophysics: An Introduction (WileyVCH, Weinheim, 2011).

[8] D. W. Jansen and C. G. de Vries, Rev. Econ. Stat. 73, 18 (1991).

[9] T. Lux, Appl. Finan. Econ. 6, 463 (1996).

[10] V. Plerou, P. Gopikrishnan, L. A. N. Amaral, M. Meyer and H. E. Stanley, Phys. Rev. E 60, 6519 (1999).

[11] R. K. Pan and S. Sinha, EPL 77, 58004 (2007).

[12] P. Gopikrishnan, M. Meyer, L. N. Amaral and H. E. Stanley, Eur. Phys. J. B 3, 139 (1998).

[13] R. K. Pan and S. Sinha, Physica A 3872055 (2008).

[14] C. G. de Vries, in F. van der Ploeg (ed.) The Handbook of International Macroeconomics (Blackwell, Oxford, 1994), p. 348.

[15] R. Friedrich, J. Peinke, and Ch. Renner, Phys. Rev. Lett. 84, 5224 (2000).

[16] J. W. McFarland, R. Richardson Pettit and S. K. Sung, J. Finance 37, 693 (1982).

[17] K. G. Koedijk, M. A. Schafgans and C. G. de Vries, J. Int. Econ. 29, 93 (1990).

[18] M. Loretan and P. C. B. Phillips, J. Empirical Finance 1, 211 (1994).

[19] D. M. Guillaume, M. M. Dacorogna, R. R. Dave, U. A. Muller, R. B. Olsen and O. V. Pictet, Finance Stochast. 1, 95 (1997).

[20] http://www.oanda.com/currency/historical-rates.

[21] E. Papaioannou, R. Portes and G. Siourounis, J. Jpn. Int. Econ. 20, 508 (2006).

[22] IMF Annual Report, 2013, Appendix I: International Reserves.

[23] http://www.xe.com/currency.

[24] G. Marsaglia, W. W. Tsang and J. Wang, J. Stat. Softw.
8, 1 (2003).

[25] J. D. Gibbons and S. Chakraborti, Nonparametric Statistical Inference, 5th Ed. (CRC Press, Boca Raton FL, 2011).

[26] http://www.msci.com/market-classification

[27] D. Coyle, GDP: A brief but affectionate history (Princeton Univ. Press, Princeton NJ, 2014).

[28] http://www.imf.org/external/pubs/ft/weo/2013/01/ weodata/index.aspx

[29] H. Theil, Economics and Information Theory (North Holland, Amsterdam, 1967).

[30] https://atlas.media.mit.edu/about/data/sources/

[31] Exchange rates of currencies of highly developed economies may on rare occasions show an extremely large deviation, e.g., that exhibited by CHF following its depegging with EUR in January 2015. However, the resulting increase in the kurtosis (e.g., for CHF, from 9.0 for the period 1995-2012 to 31.3 when the period upto 2016 is considered) is still relatively small compared to the kurtosis observed for currencies of frontier economies.

[32] A. Clauset, C. R. Shalizi and M. E. J. Newman, SIAM Rev. 51, 661 (2009).

[33] M. E. J. Newman, Contemp. Phys. 46, 323 (2005).

[34] D. A. Belsley, E. Kuh and R. E. Welsch, Regression Diagnostics, (John Wiley \& Sons, Hoboken, 1980).

[35] C.-K. Peng, S. V. Buldyrev, S. Havlin, M. Simons, H. E. Stanley and A. L. Goldberger, Phys. Rev. E 49, 1685 (1994).

[36] M. Ausloos, Physica A 285, 48 (2000).

[37] A. W. Lo and A. C. MacKinlay, J. Econometrics 40, 203 (1989).

[38] B. Brown, What Drives Global Capital Flows ? (Palgrave Macmillan, New York, 2006).

[39] S. Drozdz, J. Kwapien, F. Gruemmer, F. Ruf and J. Speth, Acta Physica Polonica B 34, 4293 (2003).

[40] S. Easwaran, M. Dixit and S. Sinha, in F. Abergel et al (eds.) Econophysics and Data Driven Modelling of Market Dynamics (Springer, Cham, 2015), p. 121.

[41] S. V. Vikram and S. Sinha, Phys. Rev. E 83, 016101 (2011).

[42] L. Feng, B. Li, B. Podobnik, T. Preis and H. E. Stanley, Proc. Natl. Acad. Sci. USA 109, 8388 (2012). 